\title{
Essentials in intraoperative indocyanine green videoangiography assessment for intracranial aneurysm surgery: conclusions from 295 consecutively clipped aneurysms and review of the literature
}

\author{
*Karl Roessler, M.D., Ph.D., ${ }^{1}$ Maximilian Krawagna, M.D., ${ }^{1}$ \\ Arnd Dörfler, M.D., Ph.D., ${ }^{2}$ Michael Buchfelder, M.D., Ph.D., 1 \\ and Oliver Ganslandt, M.D., Ph.D. ${ }^{1}$ \\ Departments of ${ }^{I}$ Neurosurgery and ${ }^{2}$ Neuroradiology, University Hospital Erlangen-Nuremberg, Erlangen, \\ Germany
}

Object. Indocyanine green (ICG) videoangiography (VA) in cerebral aneurysm surgery allows confirmation of blood flow in parent, branching, and perforating vessels as well as assessment of remnant aneurysm parts after clip application. A retrospective analysis and review of the literature were conducted to determine the current essential advantages of ICG-VA in aneurysm surgery.

Methods. The authors retrospectively evaluated all aneurysm cases treated with the aid of intraoperative ICG-VA at a single institution between 2007 and 2013. They also analyzed the literature published since the initial description of ICG-VA in 2003.

Results. Two hundred forty-six procedures were performed in 232 patients harboring 295 aneurysms. The patients, whose mean age was 54 years, consisted of 159 women and 73 men. One hundred twenty-four surgeries were performed after subarachnoid hemorrhage, and 122 were performed for incidental aneurysms. Single aneurysms were clipped in 185 patients, and multiple aneurysms were clipped in 47 (mean aneurysm diameter $6.9 \mathrm{~mm}$, range 2-40 $\mathrm{mm})$. No complications associated with ICG-VA occurred. Intraoperative microvascular Doppler ultrasonography was performed before ICG-VA in all patients, and postoperative digital subtraction angiography (DSA) studies were available in 121 patients (52.2\%) for retrospective comparative analysis. In 22 (9\%) of 246 procedures, the clip position was modified intraoperatively as a consequence of ICG-VA. Stenosis of the parent vessels (16 procedures) or occlusion of the perforators ( 6 procedures), not detected by micro-Doppler ultrasonography, were the most common problems demonstrated on ICG-VA. In another 11 procedures $(4.5 \%)$, residual perfusion of the aneurysm was observed and one or more additional clips were applied. Vessel stenosis or a compromised perforating artery occurred independent of aneurysm location and was about equally common in middle cerebral artery and anterior communicating artery aneurysms. In 2 procedures $(0.8 \%)$, aneurysm puncture revealed residual blood flow within the lesion, which had not been detected by the ICG-VA. In the postoperative DSA studies, unexpected small $(<2 \mathrm{~mm})$ aneurysm neck remnants, which had not been detected on intraoperative ICG-VA, were found in $11(9.1 \%)$ of 121 patients. However, these remnants remained without consequence except in 1 patient with a 6-mm residual aneurysm dome, which was subsequently embolized with coils.

Conclusions. In a large cohort of consecutive patients, ICG-VA proved to be a helpful intraoperative tool and led to a significant intraoperative clip modification rate of $15 \%$. However, small, $<2$-mm-wide neck remnants and a 6-mm residual aneurysm were missed by intraoperative ICG-VA in up to $10 \%$ of patients. Results in this study confirm that DSA is indispensable for postoperative quality assessment in complex aneurysm surgery. (http://thejns.org/doi/abs/10.3171/2013.11.FOCUS13475)

\section{KeY WORDS — intracranial aneurysm surgery $\quad$ • ICG videoangiography $\quad$ • aneurysm remnant $\quad$ digital subtraction angiography $\bullet$ intraoperative assessment $\bullet$ parent vessel occlusion $\quad$ perforator occlusion}

\footnotetext{
Abbreviations used in this paper: $\mathrm{ACoA}=$ anterior communicating artery; DSA = digital subtraction angiography; ICA = internal carotid artery; ICG-VA = indocyanine green videoangiography; $\mathrm{MCA}=$ middle cerebral artery; $\mathrm{PA}=$ pericallosal artery; $\mathrm{PCA}=$ posterior cerebral artery; PICA = posterior inferior cerebellar artery; $\mathrm{SAH}=$ subarachnoid hemorrhage

* Drs. Roessler and Krawagna contributed equally to this work.
}

A LTHOUGH the highest technical standards are available in most of the neurosurgical centers specialized in vascular neurosurgery all over the world, the percentage of aneurysm remnants after surgical intervention seems to be relatively constant at between $4 \%$ and $8 \%$, as demonstrated in large patient series from specialized centers. ${ }^{2,4,21}$ The rebleeding risk of aneurysm 


\section{K. Roessler et al.}

remnants (approximately 4\%), ${ }^{4}$ as well as the confidence of patients in a surgical solution for their incidental aneurysms, anticipates the need for a tool similar to digital subtraction angiography (DSA) during the endovascular procedure to avoid incomplete occlusion of a clipped cerebral aneurysm or occlusion of parent, branching, or perforating arteries causing subsequent ischemia. Intraoperative DSA would be one option to reduce the likelihood of these problems, but it is relatively laborious and time-consuming, is not available in every neurovascular surgical unit, and may add other risks (as high as $2 \%-3 \%$ ) to the patient. ${ }^{13}$ Additionally, intraoperative DSA cannot demonstrate occlusion of small perforating arteries, which can imperceptibly occur during aneurysm clipping in nearly all of the common locations ${ }^{13}$ As far back as 1967, William Feindel developed fluorescence cerebral angiography at the Montreal Neurological Institute, ${ }^{3}$ and Wrobel et al. started to use it for the intraoperative assessment of aneurysm clip placement in 1994. ${ }^{24}$ Both had worked with complex and inconvenient camera and filter systems. In 2003, Raabe et al. reported the use of indocyanine green videoangiography (ICG-VA) to assess parent, branching, and perforator vessel patency as well as complete sac occlusion during clip application in intracranial aneurysm surgery performed using the operating microscope. ${ }^{18}$ During illumination of the operating field using a near-infrared light source via the operating microscope, ICG is intravenously injected and visualized within the exposed vessels when patent. Since 2003, many investigators have reported several benefits and disadvantages of the method in its widespread applications. Even comparisons with intraoperative angiography have been made, ${ }^{23}$ but reports about the application of ICG-VA in large cohorts of consecutive patients are still rare. Thus, we analyzed a large, consecutive aneurysm case series surgically treated with the aid of ICG-VA over the last 5 years and compared our findings with reports in the literature.

\section{Methods}

\section{Patient Population}

We retrospectively searched our database for all aneurysm cases treated with the aid of intraoperative ICGVA between 2007 and 2013.

\section{Indocyanine Green Videoangiography and Postoperative DSA}

The technical principles of ICG-VA have been described in detail by Raabe et al..$^{18-20}$ For ICG-VA, a bolus of $25 \mathrm{mg}$ of ICG dye (ICG-PULSION, PULSION Medical Systems) was injected via a peripheral vein. The cost of $25 \mathrm{mg}$ of dye for one investigation in Germany is approximately $50 €$. For visualization, the Zeiss Pentero operating microscope (Carl Zeiss $\mathrm{GmbH})$ with integrated near-infrared ICG angiography (IR800) was used. Intraoperative ICG-VA was performed in all patients without complications. Intraoperative microvascular Doppler ultrasonography examinations were performed immediately before ICG-VA in all patients for testing the patency of parent and distal vessels after clip application, as well as that of the perforating artery. In cases in which parent or branching vessel stenosis or residual aneurysm necks were identified with ICG-VA, the clip position was changed or additional clips were applied and another ICG-VA was performed. Additionally, the clipped aneurysm dome was opened with a fine needle or with scissors in every patient to screen for incomplete clipping. Postoperative DSA was performed in patients when the surgeon was not absolutely sure that the aneurysm was completely occluded. Thus, a selection bias may be added for patients having postoperative angiograms for comparison and may overrepresent the group with incompletely clipped aneurysms despite negative ICG-VA. At the time of data analysis for this study, postoperative DSA was available in $121 \mathrm{pa}-$ tients $(52.2 \%)$ and retrospectively compared with the intraoperative ICG-VA.

\section{Literature Analysis}

A PubMed search was conducted using the key words "ICG," "angiography," "intracranial," and "aneurysm" alone or together.

\section{Results}

We investigated 246 consecutive procedures in 232 patients harboring 295 aneurysms surgically treated at our institution with the aid of intraoperative ICG-VA. Patient demographics are given in Table 1 . There were 159 women and 73 men with a mean age of $54 \pm 10.4$ years (range 16-79 years) with a male/female ratio of 1:2. In 124 procedures, the aneurysm was clipped after acute subarachnoid hemorrhage (SAH); in 122 procedures, incidental aneurysms were treated surgically. During 185 procedures a single aneurysm was clipped: 68 lesions at the anterior communication artery (ACoA), 91 at the middle cerebral artery (MCA), 1 at the pericallosal artery (PA), 16 at the internal carotid artery (ICA), 7 at the posterior cerebral artery (PCA), and 2 at the posterior inferior cerebellar artery (PICA). Furthermore, 47 patients were treated in 61 clipping procedures for multiple aneurysms (110 altogether). The mean diameter of the clipped aneurysms was $6.9 \mathrm{~mm}$ (range 2-40 $\mathrm{mm}$ ). In patients with multiple aneurysms, significantly more MCA and ICA and fewer ACoA aneurysms were detected, as compared with the number in patients harboring single aneurysms ( $p<0.05$, Student t-test). Altogether, 446 clips were applied, a mean of 1.6 clips per aneurysm in those with a single aneurysm and a mean of 1.5 clips per aneurysm in those with multiple aneurysms.

After performing intraoperative ICG-VA, the clip position was modified in $22(9 \%)$ of the 246 procedures to avoid ischemia, because of stenosis of the parent artery (16 procedures) or occlusion of the perforators (6 procedures), which had not been detected using the microDoppler ultrasonography. The second ICG-VA was interpreted as satisfactory after clip modification in all cases. Additionally, in another 11 procedures (4.5\%), residual perfusion of an aneurysm dome was recognized, and one or more additional clips were applied. The clipped aneurysm dome was punctured in every patient. In 2 cas- 
ICG angiography in aneurysm surgery in 246 consecutive procedures

TABLE 1: Patient demographics and summary of results

\begin{tabular}{|c|c|}
\hline Parameter & No. \\
\hline no. of procedures & 246 \\
\hline no. of patients & 232 \\
\hline males & 73 \\
\hline females & 159 \\
\hline male/female ratio & $1: 2$ \\
\hline mean age in yrs (range) & $54 \pm 10.4(16-79)$ \\
\hline no. of patients w/ SAH & 124 \\
\hline no. of patients w/ incidental aneurysms & 122 \\
\hline no. of patients w/ single aneurysms (\%) ${ }^{*}$ & 185 \\
\hline $\mathrm{ACoA}$ & $68(36.8 \%)$ \\
\hline MCA & $91(49.2 \%)$ \\
\hline ICA & $16(8.6 \%)$ \\
\hline PCA & $7(3.8 \%)$ \\
\hline PA & $1(0.5 \%)$ \\
\hline PICA & $2(1.1 \%)$ \\
\hline patients w/ multiple aneurysms $\dagger$ & 47 \\
\hline $\mathrm{ACoA}$ & $19(17.3 \%)$ \\
\hline MCA & $65(59.1 \%)$ \\
\hline ICA & $20(18.2 \%)$ \\
\hline PCA & $1(0.9 \%)$ \\
\hline PA & $5(4.5 \%)$ \\
\hline PICA & 0 \\
\hline clip adjustment rate (no. of procedures) $\ddagger$ & $9.1 \%(22)$ \\
\hline due to parent vessel occlusion & $6.6 \%(16)$ \\
\hline due to perforator occlusion & $2.5 \%(6)$ \\
\hline $\begin{array}{l}\text { rate of residual aneurysms on postop DSA } \\
\text { (no. of procedures)§ }\end{array}$ & $9.1 \%(11)$ \\
\hline \multicolumn{2}{|c|}{$\begin{array}{l}\text { * One hundred eighty-five procedures for } 185 \text { aneurysms. } \\
\dagger \text { Sixty-one procedures for } 110 \text { aneurysms. } \\
\text { † Among a total of } 242 \text { procedures. } \\
\text { § Among } 121 \text { patients. }\end{array}$} \\
\hline
\end{tabular}

es $(0.8 \%)$, hemorrhage from the sac was observed after puncture, although the sac was not filled with dye during ICG-VA (false-negative cases). Missed vessel stenosis or a compromised perforating artery after intraoperative ICGVA appeared independent of aneurysm location and was about equally common in MCA and ACoA aneurysms. Among the 121 patients in whom postoperative DSA studies were available and compared with intraoperative ICG-VA, unexpected aneurysm remnants were found in $11(9.1 \%)$. In 10 of these patients no treatment was administered because the remnants were small $(<2 \mathrm{~mm})$. However, a 6-mm residual aneurysm was detected in 1 patient after rebleeding 12 days after surgery, although the intraoperative ICG-VA indicated no remnant sac (Fig. $1 \mathrm{~F})$. This patient's residual aneurysm was identified and subsequently embolized with coils. Image quality did not differ in deep versus superficial locations, and no significant difference was found in the clip adjustment rate in the most commonly investigated sites, that is, the ACoA compared with the MCA.

\section{Summary of Literature Findings}

We identified 42 papers published since Raabe's 2003 description of ICG-VA. Thirteen studies were identified (3 prospective, 10 retrospective), which included statistical data concerning the clip adjustment rate and the rate of parent vessel occlusion or perforator impairment identified on ICG-VA after clip application (Table 2). The number of patients included in these studies varied between 14 and 190. The rate of clip adjustment as a consequence of ICG-VA results was between $2 \%$ and $38 \%$. Aneurysm remnants were identified in $2 \%-61 \%$ of cases using intraarterial DSA intraoperatively or postoperatively after ICG-VA. Perforator occlusion rates identified after ICG-VA ranged between $2 \%$ and $8.1 \%$. In our large consecutive series of 246 clipping procedures in which we performed intraoperative ICG-VA during cerebral aneurysm surgery, the aneurysm clips had to be repositioned in $9 \%$ of the procedures because of clip-associated parent vessel occlusion or occlusion of adjacent perforating arteries even though these issues were not visible using micro-Doppler ultrasonography. Additionally, in $4.5 \%$ of the procedures, one or more clips were applied because residual perfusion within the aneurysm dome or neck was detected by ICG-VA. Moreover, postoperative DSA demonstrated unexpected residual aneurysms at the neck region in $9.1 \%$ of successful clip applications guided by intraoperative ICG-VA. All residual aneurysms except one were small $(<2 \mathrm{~mm})$, and a large $6-\mathrm{mm}$ residual aneurysm led to early rehemorrhage and needed emergency retreatment.

\section{Discussion}

\section{Clip Adjustment and Residual Aneurysm Rates}

Indocyanine green videoangiography was described by Raabe et al. as a technique for intraoperative real-time analysis of blood flow within cerebral arteries and veins to assess vessel reconstruction during and after intracranial aneurysm clipping. ${ }^{18}$ In recent years, collateral circulation patency was successfully assessed using this technique during surgery for complex vascular and neoplastic lesions or during extracranial-intracranial bypass surgery. ${ }^{6,10,15}$ By applying ICG-VA during aneurysm surgery, parent vessel stenosis or occlusions of small perforating arteries can be detected and resolved in a period of about $3-5$ minutes by replacing the clips during surgery. Therefore, postoperative ischemic deficits could be reduced., ${ }^{9,20}$ Hence, in 2007 at our institution we started to routinely use ICG-VA during surgery for cerebral aneurysms along with intraoperative micro-Doppler examination.

Generally, highly variable clip adjustment rates of 2\%-38\% following ICG-VA have been reported since the introduction of this imaging technique (Table 2)., ${ }^{1,5,9}$ $16,18,20,22,23$ Obviously, this high variance in the intraoperative benefit rate may be attributable to a low number of patients, inhomogeneous patient groups, and the nonsystematic application of the technique in previous studies. Thus, the general benefit of ICG-VA in aneurysm surgery is still under discussion. Although many detailed reports about ICG-VA application in cerebral aneurysm surgery 


\section{K. Roessler et al.}
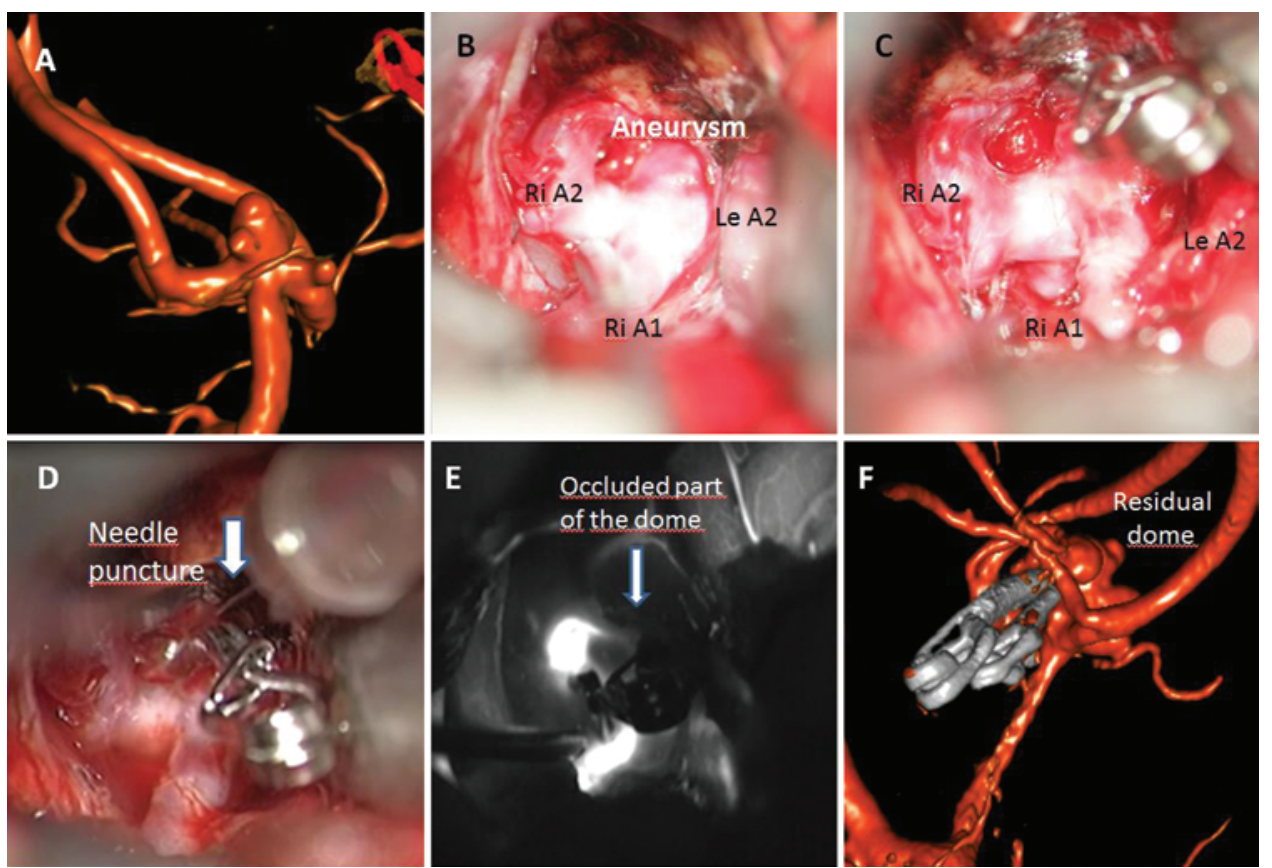

FIG. 1. A: A 47-year-old male presented with a Hunt and Hess Grade II SAH. Digital subtraction angiography revealed an ACoA aneurysm $7 \mathrm{~mm}$ in diameter, as well as a small aneurysm on the left $A_{2}$. B: Intraoperative view of the aneurysm and the parent and branching vessels. $\quad$ C and D: The dome was occluded with 2 clips, and puncture with a fine needle revealed no residual blood flow. E: The ICG-VA demonstrated sufficient flow in parent and branching vessels, and no signs of residual dome parts were recognized. F: The patient presented with clinical and tomographic rehemorrhage on Day 12 postsurgery, and DSA revealed a significant residual aneurysm dome, which was embolized with coils during the same imaging procedure. $A 1=$ $\mathrm{A}_{1}$ segment; $\mathrm{A} 2$ = $\mathrm{A}_{2}$ segment; $\mathrm{Ri}=$ right; Le = left.

exist, some points may need clarification since hospital economists or neurosurgical colleagues regularly question whether postoperative DSA should be performed if the intraoperative ICG-VA is uneventful. Analysis of our large collective of 232 consecutive patients and the systematic application of the technique revealed some valuable information: We found a $9 \%$ rate of clip replacement due to parent vessel stenosis or perforator occlusion and a $4.5 \%$ rate of residual perfusion of the aneurysm dome or neck leading to additional clip application. Therefore, we concluded that nearly every sixth patient profits from the application of this technique when routinely used during cerebral aneurysm surgery. Moreover, we demonstrated that ICG-VA is more accurate than neurovascular Dop-

TABLE 2: Literature summary of intraoperative clip adjustment rates due to ICG-VA*

\begin{tabular}{lccc}
\hline \multicolumn{1}{c}{ Authors \& Year } & No. of Patients Undergoing ICG-VA & Clip Adjustment Rate & \% Aneurysm Remnants \\
\hline Raabe et al., 2003 & 14 & 7.1 & NA \\
Raabe et al., 2005 & 114 & 9 & NA \\
Imizu et al., 2008 & 13 & 38 & NA \\
Li et al., 2009 & 120 & 4.6 & 6.7 \\
Ma et al., 2009 & 45 & 4.4 & 6 \\
Dashti et al., 2009 & 190 & 12 & 2 \\
Jing et al., 2010 & 42 & 5 & NA \\
Wang et al., 2010 & 129 & 2 & 6 \\
Fischer et al., 2010 & 50 & 10 & $\mathrm{NA}$ \\
Gruber et al., 2011 & 104 & 6.7 & 10 \\
Oda et al., 2011 & 39 & 10 & 6.1 \\
Washington et al., 2013 & 49 & 4.1 & 3.6 \\
Özgiray et al., 2013 & 109 & 1.8 & 4.7 \\
Moon et al., 2013 & 127 & 6.3 & 4.5 \\
current study & 232 & 9.1 & \\
\hline
\end{tabular}

* NA = not applicable. 


\section{ICG angiography in aneurysm surgery in 246 consecutive procedures}

pler ultrasonography, because all patients who needed clip replacement after ICG-VA had had unremarkable Doppler ultrasonography. Our rate of $10 \%-15 \%$ of patients in whom the clip position was changed due to parent vessel stenosis, perforator occlusion, or incomplete dome occlusion seems reliable, as many studies with high patient numbers, especially the prospective ones, have reported similar results. . $^{1,5,16,20}$

\section{Indocyanine Green Angiography and Intraoperative and Postoperative DSA}

Recently, the technique of ICG-VA was compared with intraoperative DSA. ${ }^{19,23}$ But some main differences in the two techniques make a direct comparison unfeasible. In ICG-VA, only the dissected and thus visible vascular structures within the operative field can be evaluated. ${ }^{18}$ Additionally, in ICG-VA, the view can be obstructed by arteriosclerotic or calcified plaques within the vessel walls or simply by cisternal blood after $\mathrm{SAH},{ }^{7,20}$ which makes accurate interpretation impossible. Compared with DSA, even multiple ICG angiographic studies within one procedure are inexpensive and easy to perform and carry a very low risk of complications. No additional time-consuming procedure interrupting the surgery for catheter replacements under fluoroscopic guidance is necessary. ${ }^{24} \mathrm{In}$ contrast to intraoperative DSA, ICG-VA can excellently identify perforating arteries. ${ }^{20}$ Nevertheless, intraoperative DSA remains the most reliable intraoperative tool for excluding an incompletely clipped cerebral aneurysm.,23

One of the main advantages of DSA over ICG-VA seems to be easier identification of the aneurysm neck remnant. ${ }^{9,23}$ This fact is also confirmed by our current study. In $9.1 \%$ of the patients, neck remnants not intraoperatively detected with ICG-VA were identified on postoperative DSA. We saw this as the main drawback of ICG-VA and led to close observation of a patient group with residual aneurysms, which would not have been necessary when we used intraoperative DSA. Even one case of misclipping an ACoA aneurysm occurred in our series, which was not detected during ICG-VA and surprisingly appeared as a 6-mm dome rest perfusion in the postoperative DSA after early rebleeding (Fig. 1). The main issue leading to this significant difference may be the inability of ICG-VA to visualize hidden parts of the parent, branching, and perforator vessels as well as undissected parts of the aneurysm dome, especially after one's ability to manipulate is inhibited by the applied aneurysm clips. Indocyanine green videoangiography seems reliable only if the complete vascular anatomy is properly dissected and the clipped aneurysm is visualized completely. These essential points seem to explain the significant discordance of up to $15 \%$ in reported rates between the two modalities. ${ }^{23}$ As a consequence, at least after clipping complex aneurysms, postoperative DSA remains the gold standard for the evaluation of treatment success (Table 3).

\section{Aneurysm Location and ICG-VA Clip Adjustment Rate}

In the literature review, deeper aneurysm locations, as in ACoA aneurysms compared with MCA aneurysms, seem to further diminish the benefits of ICG-VA. ${ }^{7,9} \mathrm{We}$ could not confirm this finding in our series. Image quality did not differ for deep versus superficial locations; thus, no significant difference was found in the clip adjustment rate in the most commonly investigated sites, the ACoA compared with the MCA location.

\section{Value of ICG-VA as a Substitute for Postoperative DSA}

In a comparison of clip adjustment rates in our series with rates from intraoperative DSA reports, intraoperative DSA proved twice as sensitive as ICG-VA. ${ }^{19,23}$ Given this finding together with neck remnant rates of up to $10 \%$ with ICG-VA, ${ }^{16}$ postoperative DSA seems indispensable for quality assurance in complex cerebral aneurysm surgery; especially in complex vascular anatomy, intraoperative ICG-VA cannot visualize all parts of the operating field after clip application. Another weakness of ICG-VA seems to be a low intravascular flow area, especially with insufficiently clipped aneurysm domes, as we found in 2 cases with clipped aneurysm domes that registered negative on ICG-VA; surprisingly, these domes bled after intraoperative needle puncture. A small residual orifice in the neck area seems to be detected only by opening the aneurysm dome and allowing free blood flow, but it is overlooked by ICG-VA because the dye cannot penetrate a small opening against the pressure of the blood within the aneurysm dome. Although such cases naturally have a high likelihood of occluding over time via thrombosis, this case nevertheless demonstrates a possible weakness of the method.

\section{Strengths and Limitations of the Study}

One of the most valuable strengths of the study is the high number of patients, who were consecutively surgically treated using the ICG-VA technique. This enabled us to gain a reliable sensitivity of the method in detecting potential dangerous complications during clipping of cerebral aneurysms. In our study, we found a stable rate of $15 \%$ for detecting compromised parent vessels or perforators or incomplete neck occlusions. On the other hand, one limitation of our study is the fact that a prospective comparison with intraoperative DSA is lacking.

\section{Conclusions}

Nevertheless, our data suggest that ICG-VA is a safe and easy-to-perform intraoperative method for evaluating compromised intravascular circulation within small or large parent and branching vessels as well as the occluded dome or neck of a cerebral aneurysm during surgery. Thus, the very low complication rate, the low costs of the technique, and the described benefit rate of nearly $15 \%$ of the procedures argue for routine application during clipping of cerebral aneurysms. In contrast, in up to $10 \%$ of patients, small aneurysm sac remnants can be overlooked intraoperatively by ICG-VA. In most cases, these small residual aneurysms may not have consequences for the immediate postoperative course but may give rise to recurrent aneurysms in the long term. We highly recommend postoperative DSA to look for significant errors in aneurysm clipping, even if intraoperative ICG-VA has 
K. Roessler et al.

TABLE 3: Essential advantages and disadvantages of ICG-VA*

\begin{tabular}{ll}
\hline \multicolumn{1}{c}{ Advantages } & \multicolumn{1}{c}{ Disadvantages } \\
\hline $\begin{array}{l}\text { avoidance of vessel stenosis or incomplete aneurysm occlusion } \\
\text { in about 15\% of cases }\end{array}$ & $\begin{array}{l}\text { not reliable in complex aneurysm anatomy, substitution of DSA } \\
\text { not possible } \\
\text { only dissected vessels are assessable } \\
\text { short performance time (3-5 minutes) }\end{array} 60^{\circ}$ view \\
low costs compared w/ DSA (one study costs about $50 €)$ & plaques \& blood obscure the assessment \\
very low complication rate (0.0019\% risk of SAEs) & incorrect during evaluation of intravascular low-flow sectors \\
repetitive assessments during 1 surgery possible & false-negative assessment possible \\
clip replacement w/in ischemic threshold time possible & no 3D reconstruction available \\
short interruption of surgical action & \\
perforator patency inspection reliable & \\
surgical manipulation during ICG-VA possible & \\
\hline
\end{tabular}

* $\mathrm{SAE}=$ severe adverse event.

been performed and especially after clipping complex aneurysms.

\section{Disclosure}

The authors report no conflict of interest concerning the materials or methods used in this study or the findings specified in this paper.

Author contributions to the study and manuscript preparation include the following. Conception and design: Roessler. Acquisition of data: Krawagna, Dörfler. Analysis and interpretation of data: Roessler, Krawagna, Ganslandt. Drafting the article: Roessler. Critically revising the article: all authors. Approved the final version of the manuscript on behalf of all authors: Roessler. Administrative/ technical/material support: Roessler. Study supervision: Roessler, Buchfelder.

\section{References}

1. Dashti R, Laakso A, Niemelä M, Porras M, Hernesniemi J: Microscope-integrated near-infrared indocyanine green videoangiography during surgery of intracranial aneurysms: the Helsinki experience. Surg Neurol 71:543-550, 2009

2. David CA, Vishteh AG, Spetzler RF, Lemole M, Lawton MT, Partovi S: Late angiographic follow-up review of surgically treated aneurysms. J Neurosurg 91:396-401, 1999

3. Feindel W, Yamamoto YL, Hodge CP: Intracarotid fluorescein angiography: a new method for examination of the epicerebral circulation in man. Can Med Assoc J 96:1-7, 1967

4. Feuerberg I, Lindquist C, Lindqvist M, Steiner L: Natural history of postoperative aneurysm rests. J Neurosurg 66:30-34, 1987

5. Fischer G, Stadie A, Oertel JM: Near-infrared indocyanine green videoangiography versus microvascular Doppler sonography in aneurysm surgery. Acta Neurochir (Wien) 152: 1519-1525, 2010

6. Gruber A, Dorfer C, Bavinzski G, Standhardt H, Ferraz-Leite $\mathrm{H}$, Knosp E: Superselective indocyanine green angiography for selective revascularization in the management of peripheral cerebral aneurysms. AJNR Am J Neuroradiol 33:E36E37, 2012

7. Gruber A, Dorfer C, Standhardt H, Bavinzski G, Knosp E: Prospective comparison of intraoperative vascular monitoring technologies during cerebral aneurysm surgery. Neurosurgery 68:657-673, 2011

8. Imizu S, Kato Y, Sangli A, Oguri D, Sano H: Assessment of incomplete clipping of aneurysms intraoperatively by a nearinfrared indocyanine green-video angiography (Niicg-Va) in- tegrated microscope. Minim Invasive Neurosurg 51:199-203, 2008

9. Jing Z, Ou S, Ban Y, Tong Z, Wang Y: Intraoperative assessment of anterior circulation aneurysms using the indocyanine green video angiography technique. J Clin Neurosci 17:2628, 2010

10. Kim DL, Cohen-Gadol AA: Indocyanine-green videoangiogram to assess collateral circulation before arterial sacrifice for management of complex vascular and neoplastic lesions: technical note. World Neurosurg 79:404.e1-404.e6, 2013

11. Li J, Lan Z, He M, You C: Assessment of microscope-integrated indocyanine green angiography during intracranial aneurysm surgery: a retrospective study of 120 patients. Neurol India 57:453-459, 2009

12. Ma CY, Shi JX, Wang HD, Hang CH, Cheng HL, Wu W: Intraoperative indocyanine green angiography in intracranial aneurysm surgery: Microsurgical clipping and revascularization. Clin Neurol Neurosurg 111:840-846, 2009

13. Martin NA, Bentson J, Viñuela F, Hieshima G, Reicher M, Black K, et al: Intraoperative digital subtraction angiography and the surgical treatment of intracranial aneurysms and vascular malformations. J Neurosurg 73:526-533, 1990

14. Moon HS, Joo SP, Seo BR, Jang JW, Kim JH, Kim TS: Value of indocyanine green videoangiography in deciding the completeness of cerebrovascular surgery. J Korean Neurosurg Soc 53:349-355, 2013

15. Murai Y, Adachi K, Takagi R, Koketsu K, Matano F, Teramoto A: Intraoperative Matas test using microscope-integrated intraoperative indocyanine green videoangiography with temporary unilateral occlusion of the A1 segment of the anterior cerebral artery. World Neurosurg 76:477.e7-477.e10, 2011

16. Oda J, Kato Y, Chen SF, Sodhiya P, Watabe T, Imizu S, et al: Intraoperative near-infrared indocyanine green-videoangiography (ICG-VA) and graphic analysis of fluorescence intensity in cerebral aneurysm surgery. J Clin Neurosci 18:1097-1100, 2011

17. Özgiray E, Aktüre E, Patel N, Baggott C, Bozkurt M, Niemann D, et al: How reliable and accurate is indocyanine green video angiography in the evaluation of aneurysm obliteration? Clin Neurol Neurosurg 115:870-878, 2013

18. Raabe A, Beck J, Gerlach R, Zimmermann M, Seifert V: Nearinfrared indocyanine green video angiography: a new method for intraoperative assessment of vascular flow. Neurosurgery 52:132-139, 2003

19. Raabe A, Beck J, Seifert V: Technique and image quality of intraoperative indocyanine green angiography during aneurysm surgery using surgical microscope integrated near-infrared video technology. Zentralbl Neurochir 66:1-8, 2005 


\section{ICG angiography in aneurysm surgery in 246 consecutive procedures}

20. Raabe A, Nakaji P, Beck J, Kim LJ, Hsu FP, Kamerman JD, et al: Prospective evaluation of surgical microscope-integrated intraoperative near-infrared indocyanine green videoangiography during aneurysm surgery. J Neurosurg 103:982-989, 2005

21. Sindou M, Acevedo JC, Turjman F: Aneurysmal remnants after microsurgical clipping: classification and results from a prospective angiographic study (in a consecutive series of 305 operated intracranial aneurysms). Acta Neurochir (Wien) 140:1153-1159, 1998

22. Wang S, Liu L, Zhao Y, Zhang D, Yang M, Zhao J: Evaluation of surgical microscope-integrated intraoperative near-infrared indocyanine green videoangiography during aneurysm surgery. Neurosurg Rev 34:209-215, 2010

23. Washington CW, Zipfel GJ, Chicoine MR, Derdeyn CP, Rich $\mathrm{KM}$, Moran CJ, et al: Comparing indocyanine green videoan- giography to the gold standard of intraoperative digital subtraction angiography used in aneurysm surgery. Clinical article. J Neurosurg 118:420-427, 2013

24. Wrobel CJ, Meltzer H, Lamond R, Alksne JF: Intraoperative assessment of aneurysm clip placement by intravenous fluorescein angiography. Neurosurgery 35:970-973, 1994

Manuscript submitted October 14, 2013.

Accepted November 18, 2013.

Please include this information when citing this paper: DOI: 10.3171/2013.11.FOCUS13475.

Address correspondence to: Karl Roessler, M.D., Ph.D., Department of Neurosurgery, University Hospital Erlangen, Schwabachanlage 6,91054 Erlangen, Germany. email: karl.roessler@uk-erlangen. de. 